\title{
Distributed Regulation Allocation with Aggregator Coordinated Electric Vehicles
}

\author{
Sun Sun*, Min Dong ${ }^{\dagger}$, and Ben Liang* \\ *Dept. of Electrical and Computer Engineering, University of Toronto, Canada \\ ${ }^{\dagger}$ Dept. of Electrical, Computer and Software Engineering, University of Ontario Institute of Technology, Canada \\ Email: \{ssun, liang\}@ comm.utoronto.ca, min.dong@uoit.ca
}

\begin{abstract}
Electric vehicles (EVs) are promising alternatives to provide ancillary services in future smart energy systems. In this paper, we consider an aggregator-EVs system providing regulation service to a power grid. To allocate regulation amount among EVs, we present both synchronous and asynchronous distributed algorithms, which align each EV's interest with the system's benefit. Compared with previous works, our algorithms accommodate a more realistic model of the aggregator-EVs system, in which EV battery degradation cost, EV charging/discharging inefficiency, EV energy gain/loss, the cost of external energy sources, and potential asynchronous communication between the aggregator and each $\mathrm{EV}$ are taken into account. We give sufficient conditions under which the proposed algorithms generate the optimal regulation amounts. Simulations are shown to validate our theoretical results.
\end{abstract}

\section{INTRODUCTION}

Frequency regulation service is provided to balance power generation and load demand in a short time scale, so as to maintain the frequency of a power grid at its nominal value. Traditionally, regulation service is provided by fast responsive generators, which vary their output to alleviate power deficits or surpluses, and is the most expensive ancillary service [1]. More recently, experiments have shown that EV's power electronics and battery can well respond to the frequent regulation signals [2]. Thus, it is possible to exploit plugged-in EV as an alternative to provide regulation service through charging and discharging, which could reduce the cost of regulation service significantly and may be more environmentally friendly [2].

However, since regulation service is generally requested on the order of megawatts while the power capacity of an EV is typically $5-20 \mathrm{~kW}$, it is often necessary for an aggregator to coordinate a large number of EVs to join the regulation market [3]. To allocate the requested regulation amount in an aggregator-EVs system, one may rely on a centralized control architecture, in which the aggregator determines the regulation amounts for all EVs. Nevertheless, letting the aggregator fully control EV charging/discharging would override the user's individual choice, hence potentially hampering the adoption of vehicle-to-grid (V2G) technology [4]. An alternative approach, which is the focus of this paper, is to distribute the decision making over EVs. In this case, it is important to design a proper distributed algorithm, in which each EV's interest can be aligned with the system's benefit.

There is a growing body of recent works on $\mathrm{V} 2 \mathrm{G}$ regulation service. Specific to the aggregator-EVs system, which focuses on the interaction between the aggregator and EVs, most works adopt centralized regulation allocation, with the objective of maximizing the profit of the aggregator or the EVs, e.g., [5]-[9], or the social welfare of the system, e.g., [10]. To our best awareness, the only previous works that address distributed regulation allocation specific to the aggregatorEVs system are [11] and [12]. Both apply a game-theoretical approach to design pricing strategies that incentivize EVs' participation. In [11], assuming that each EV only decides whether to charge, discharge, or remain idle without considering the charging/discharging amount, a service pricing function is developed leading to a Nash equilibrium, where the difference between the requested regulation amount and the sum EV contribution is minimized. The same goal is considered in [12], where each EV can additionally decide its charging/discharging amount. A pricing strategy and a distributed consensus algorithm are designed for the EVs to reach a unique Nash equilibrium, but the optimality of the pricing strategy is not considered.

In this paper, we provide both synchronous and asynchronous distributed algorithms for the aggregator-EVs system to collectively optimize the regulation allocation among selfish EVs, aligning each EV's interest with the system's benefit. We include the cost of clearing the difference between the requested regulation amount and the sum EV contribution into the optimization objective. The aggregator is assumed to be regulated and non profit-driven, so that it aims to minimize the total cost of the whole system. Compared with previous works, we also consider a more detailed model of the aggregatorEVs system. Specifically, for each EV, the battery degradation cost due to frequent charging/discharging in regulation service, charging/discharging inefficiency, and energy gain/loss, are taken into account. We study both the ideal scenario in which the aggregator and each EV can communicate synchronously without delay, and the practical scenario in which the information at both sides can be outdated. To develop distributed algorithms, we apply the technique of Lagrange dual decomposition [13], which has found wide application in network utility maximization [14]. For both synchronous and asynchronous distributed algorithms, we give sufficient conditions under which the algorithms produce the optimal regulation amounts.

The remainder of this paper is organized as follows. We describe the system model and formulate the regulation al- 


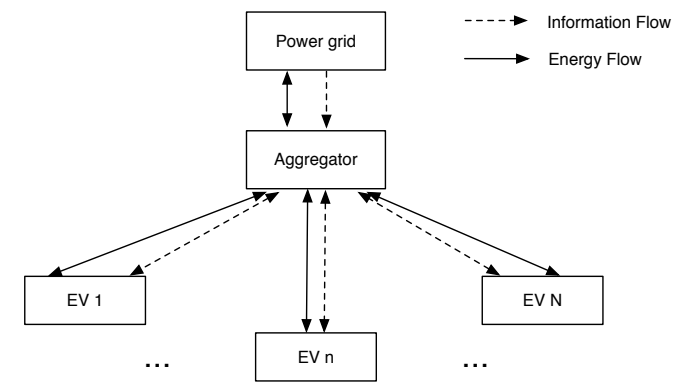

Fig. 1. Information and energy flows in the aggregator-EVs system.

location problem in Section II. In Section III, we propose a synchronous distributed algorithm for regulation allocation, and in Section IV, we provide an asynchronous distributed algorithm. Simulation results are shown in Section V, and we conclude in Section VI.

Notation: Denote $[a, b]^{-}$as $\min \{a, b\}$; denote $[x]_{a}^{b}$ as $\min \{\max \{x, a\}, b\}$, which projects $x$ onto the interval $[a, b]$; denote $\mathbb{R}$ as the set of real numbers and $\mathbb{Z}^{+}$as the set of nonnegative integers.

\section{SySTEM MOdeL}

In this section, we first describe an aggregator-EVs system which provides regulation service, then formulate the regulation allocation problem mathematically.

\section{A. Regulation Service and Aggregator-EVs System}

Consider an aggregator-EVs system in which an aggregator coordinates $N$ EVs to provide regulation service to a power grid. The regulation signal is random and is sent by the power grid to the aggregator periodically. Consider one period of regulation service, and denote $G$ as the regulation signal received by the aggregator. If $G>0$, the aggregator needs to offer regulation down service by absorbing $G$ units of energy from the power grid during the current period; if $G<0$, the aggregator needs to offer regulation up service by contributing $|G|$ units of energy to the power grid during the current period. For notation simplicity, let $\mathbf{1}_{d} \triangleq \mathbf{1}(G>0)$ and $\mathbf{1}_{u} \triangleq \mathbf{1}(G<0)$, where $\mathbf{1}(\cdot)$ is the indicator function. Since regulation down and up services cannot happen simultaneously, we have $\mathbf{1}_{d} \cdot \mathbf{1}_{u}=0$. To provide regulation service, assume that the aggregator and each EV can communicate bidirectionally using a communication protocol such as ZigBee or Bluetooth. In Fig. 1, the information and energy flows of the system are depicted.

In the considered regulation service period, denote $x_{i, d} \geq 0$ as the amount of energy absorbed by the $i$-th $\mathrm{EV}$ in the case of regulation down, and $x_{i, u} \geq 0$ as the amount of energy contributed by the $i$-th EV in the case of regulation up. Define $N$-dimensional vectors $\mathbf{x}_{d} \triangleq\left[x_{1, d}, \cdots, x_{N, d}\right]$ and $\mathbf{x}_{u} \triangleq\left[x_{1, u}, \cdots, x_{N, u}\right]$.

Let $s_{i, b}$ be the energy state of the $i$-th EV at the beginning of the current period, and $s_{i, e}$ be the energy state after regulation service. The relationship between $s_{i, b}$ and $s_{i, e}$ is as follows.

$$
s_{i, e}=s_{i, b}+\mathbf{1}_{d} \eta_{i, \mathrm{ch}} x_{i, d}-\mathbf{1}_{u} \eta_{i, \mathrm{di}} x_{i, u},
$$

where $\eta_{i, \text { ch }} \in(0,1]$ is the charging efficiency coefficient, and $\eta_{i, \mathrm{di}} \geq 1$ is the discharging efficiency coefficient. Due to the inefficiency of EV charging/discharging, generally, the actual stored energy for regulation down is less than $x_{i, d}$, and the actual contributed energy for regulation up is larger than $x_{i, u}$. Because of charging/discharging circuit limitation, assume that $x_{i, d}$ and $x_{i, u}$ are upper bounded by $x_{i, \max }>0$. Charging a battery near its capacity or discharging it close to the zero energy state can significantly reduce battery lifetime [15]. Therefore, lower and upper bounds on the battery energy state are usually imposed by its manufacturer or user. Denote $s_{i \text {,cap }}$ as the energy capacity of the $i$-th $\mathrm{EV}$, and $\left[s_{i, \min }, s_{i, \max }\right]$ as its preferred energy range with $0 \leq s_{i, \min }<s_{i, \max } \leq s_{i, \text { cap }}$. By the above constraints, the regulation amounts $x_{i, d}$ and $x_{i, u}$ of the $i$-th EV should satisfy $0 \leq x_{i, d} \leq h_{i, d}$ and $0 \leq$ $x_{i, u} \leq h_{i, u}$, respectively, where $h_{i, d} \triangleq\left[x_{i, \max }, \frac{s_{i, \max }-s_{i, b}}{\eta_{i, \mathrm{ch}}}\right]^{-}$ and $h_{i, u} \triangleq\left[x_{i, \max }, \frac{s_{i, b}-s_{i, \min }}{\eta_{i, \mathrm{di}}}\right]^{-}$.

By providing regulation service, each EV receives payment from the aggregator for its controllable and flexible charging/discharging capability. Denote the unit prices of regulation down and up for the current period as $p_{d}$ and $p_{u}$, respectively. Then, the $i$-th EV gets payment $p_{d} x_{i, d}$ in the case of regulation down and payment $p_{u} x_{i, u}$ in the case of regulation up.

However, for each EV, the regulation service gain comes at the cost of battery degradation for frequent charging and extra cycling of battery [16], [17]. Denote $C_{i, d}(\cdot)$ and $C_{i, u}(\cdot)$ as the degradation cost functions of the regulation amount associated with regulation down and up, respectively, with $C_{i, d}(0)=C_{i, u}(0)=0$. For notation simplicity, we will merge the parameter $\eta_{i, \text { di }}$ into the cost function $C_{i, u}(\cdot)$. Since faster charging or discharging (larger value of $x_{i, d}$ or $x_{i, u}$ ) generally has a disproportionally more detrimental effect on battery lifetime, $C_{i, d}(\cdot)$ and $C_{i, u}(\cdot)$ are generally increasing convex functions. To facilitate later analysis, we slightly strengthen this condition to the following:

\section{C1:}

- $C_{i, d}(x)$ (resp. $\left.C_{i, u}(x)\right)$ is increasing, strictly convex, and twice continuously differentiable on $\left[0, h_{i, d}\right]$ (resp. $\left.\left[0, h_{i, u}\right]\right)$.

- The second derivatives satisfy $C_{i, d}^{\prime \prime}(x) \geq c_{i, d}>0, \forall x \in$ $\left[0, h_{i, d}\right]$ (resp. $\left.C_{i, u}^{\prime \prime}(x) \geq c_{i, u}>0, \forall x \in\left[0, h_{i, u}\right]\right)$.

Additionally, for each EV, there is energy gain/loss associated with regulation down/up. Denote the unit market charging price as $p_{m}$. Then, for the $i$-th $\mathrm{EV}$, the revenue due to absorbing energy in the case of regulation down is $p_{m} x_{i, d}$, and the loss due to contributing energy in the case of regulation up is $p_{m} \eta_{i, \mathrm{di}} x_{i, u}$. Therefore, the effective cost of the $i$-th $\mathrm{EV}$ for providing regulation service is $\mathbf{1}_{d}\left(C_{i, d}\left(x_{i, d}\right)-p_{m} x_{i, d}-p_{d} x_{i, d}\right)+\mathbf{1}_{u}\left(C_{i, u}\left(x_{i, u}\right)\right.$ $\left.+p_{m} \eta_{i, \mathrm{di}} x_{i, u}-p_{u} x_{i, u}\right)$.

Due to a lack of participating EVs or high battery degradation cost, the total regulation amount provided by the EVs may be insufficient to meet the requested regulation amount. Specifically, for regulation down, this insufficiency 
of regulation amount means that $\sum_{i=1}^{N} x_{i, d}<G$, with the associated gap representing an energy surplus; for regulation up, this insufficiency means that $\sum_{i=1}^{N} x_{i, u}<|G|$, with the associated gap representing an energy deficit. Therefore, from time to time, to fill such gaps, the aggregator needs to exploit more expensive external energy sources, such as from the traditional regulation market. Denote the cost functions of clearing energy surplus and energy deficit as $D_{s}(\cdot)$ and $D_{d}(\cdot)$, respectively, with $D_{s}(0)=D_{d}(0)=0$. Then, the cost for the aggregator to exploit the external sources is $\mathbf{1}_{d} D_{s}\left(G-\sum_{i=1}^{N} x_{i, d}\right)+\mathbf{1}_{u} D_{d}\left(|G|-\sum_{i=1}^{N} x_{i, u}\right)$. We impose the following conditions on the external cost functions:

\section{C2:}

- $D_{s}(x)$ (resp. $\left.D_{d}(x)\right)$ is increasing, strictly convex, and twice continuously differentiable on $[0, G]$.

- The second derivatives satisfy $D_{s}^{\prime \prime}(x) \geq d_{s}>0, \forall x \in$ $[0, G]\left(\operatorname{resp} . D_{d}^{\prime \prime}(x) \geq d_{d}>0, \forall x \in[0, G]\right)$.

\section{B. Problem Statement}

Because the EVs may belong to different users other than the aggregator, we assume that each EV is selfish and intends to minimize its own effective cost given the regulation prices. In particular, for each period of regulation service, the $i$-th EV aims to solve the following optimization problem.

$$
\begin{array}{cc}
\min _{x_{i, d}, x_{i, u}} & \mathbf{1}_{d}\left(C_{i, d}\left(x_{i, d}\right)-p_{m} x_{i, d}-p_{d} x_{i, d}\right) \\
& \quad+\mathbf{1}_{u}\left(C_{i, u}\left(x_{i, u}\right)+p_{m} \eta_{i, \mathrm{di}} x_{i, u}-p_{u} x_{i, u}\right) \\
\text { s.t. } & 0 \leq x_{i, d} \leq \mathbf{1}_{d} h_{i, d}, \quad 0 \leq x_{i, u} \leq \mathbf{1}_{u} h_{i, u} .
\end{array}
$$

The aggregator is assumed to be regulated and non profitdriven, and it can represent a government-funded party that encourages the integration of EVs into a power grid. The objective of the aggregator is to minimize the total cost of the whole system in the service period. The optimization problem for the aggregator is thus as follows.

$$
\begin{aligned}
\min _{\mathbf{x}_{d}, \mathbf{x}_{u}} & \mathbf{1}_{d}\left(\left[\sum_{i=1}^{N} C_{i, d}\left(x_{i, d}\right)-p_{m} x_{i, d}\right]+D_{s}\left(G-\sum_{i=1}^{N} x_{i, d}\right)\right) \\
+\mathbf{1}_{u}( & {\left.\left[\sum_{i=1}^{N} C_{i, u}\left(x_{i, u}\right)+p_{m} \eta_{i, \mathrm{di}} x_{i, u}\right]+D_{d}\left(|G|-\sum_{i=1}^{N} x_{i, u}\right)\right) } \\
\text { s.t. } \quad & 0 \leq x_{i, d} \leq \mathbf{1}_{d} h_{i, d}, \quad 0 \leq x_{i, u} \leq \mathbf{1}_{u} h_{i, u}, \forall i, \\
& \sum_{i=1}^{N} x_{i, d} \leq G, \quad \sum_{i=1}^{N} x_{i, u} \leq|G| .
\end{aligned}
$$

In the objective function above, the EVs' payments for regulation service are not included. This is because the payments are transfered from the aggregator to the EVs, hence not affecting the cost of the whole system. The aggregator can solve the above optimization problem centrally, provided that it can fully control EV charging/discharging, and is aware of each EV's upper bounds on the regulation amounts and degradation cost functions. However, EV user may wish to keep its individual choice; plus, the required information is private to each $\mathrm{EV}$, so it may not be available at the aggregator.
Motivated by these concerns, our goal in this paper is to develop distributed algorithms in which the private information of EVs is not required, but each EV's self interest can be aligned with the system's benefit.

\section{SyNCHRONOUS DistribUtED REGULATION ALLOCATION}

Applying the technique of Lagrange dual decomposition [13], we present a distributed algorithm for regulation allocation, provided that the aggregator can communicate with EVs synchronously without delay. A more realistic asynchronous distributed algorithm is considered in Section IV.

\section{A. Synchronous Algorithm}

Since regulation down and up services cannot happen simultaneously and their analyses are similar, in the following, we focus on regulation down service only, i.e., $\mathbf{1}_{d}=1$. The optimization problem for the aggregator is as follows.

$$
\begin{aligned}
\text { P1: } \min _{\mathbf{x}_{d}} & {\left[\sum_{i=1}^{N} C_{i, d}\left(x_{i, d}\right)-p_{m} x_{i, d}\right]+D_{s}\left(G-\sum_{i=1}^{N} x_{i, d}\right) } \\
\text { s.t. } \quad & 0 \leq x_{i, d} \leq h_{i, d}, \forall i, \quad \sum_{i=1}^{N} x_{i, d} \leq G .
\end{aligned}
$$

For $\mathbf{P 1}$, since $C_{i, d}(\cdot)$ is strictly convex and thus the objective function is strictly convex, there is at most one global minimizer. Additionally, since the objective function is continuous and the constraint set of $\mathbf{x}_{d}$ is compact, there is at least one minimizer. Therefore, there is a unique solution for $\mathbf{P 1}$.

Note that the term $D_{s}\left(G-\sum_{i=1}^{N} x_{i, d}\right)$ in the objective function, as well as the constraint $\sum_{i=1}^{N} x_{i, d} \leq G$, couples the regulation amounts of all EVs, which hinders a distributed algorithm. To avoid such coupling, we first introduce an auxiliary variable $q \in[0, G]$ to represent the amount of energy surplus and consider the following problem.

$$
\begin{aligned}
\text { P2: } \min _{\mathbf{x}_{d}, q} & {\left[\sum_{i=1}^{N} C_{i, d}\left(x_{i, d}\right)-p_{m} x_{i, d}\right]+D_{s}(q) } \\
\text { s.t. } \quad & 0 \leq x_{i, d} \leq h_{i, d}, \forall i, \quad 0 \leq q \leq G, \\
& \sum_{i=1}^{N} x_{i, d}+q=G .
\end{aligned}
$$

Denote the optimal solution of $\mathbf{P 2}$ as $\left(\mathbf{x}_{d}^{*}, q^{*}\right)$ and the optimal value of $\mathbf{P 2}$ as $f^{*}$. It is not hard to see that, $\mathbf{P 2}$ has a unique optimal solution, and $\mathbf{P 2}$ and $\mathbf{P 1}$ are equivalent in the sense that these two have the same unique solution $\mathbf{x}_{d}^{*}$ and the same objective value.

Next associate the equality constraint in (1) with a Lagrange multiplier $\lambda$. The partial Lagrangian of $\mathbf{P 2}$ is

$$
\begin{aligned}
& L\left(\mathbf{x}_{d}, q, \lambda\right) \\
& =\left[\sum_{i=1}^{N} C_{i, d}\left(x_{i, d}\right)-p_{m} x_{i, d}\right]+D_{s}(q)+\lambda\left(G-\sum_{i=1}^{N} x_{i, d}-q\right) .
\end{aligned}
$$


The dual function $g(\lambda)$ is defined as the minimum of the partial Lagrangian with respect to the primal variables $\mathbf{x}_{d}$ and $q$ :

$$
g(\lambda)=\min _{0 \leq x_{i, d} \leq h_{i, d}, \forall i, 0 \leq q \leq G} L\left(\mathbf{x}_{d}, q, \lambda\right),
$$

which can be decomposed into sub-problems for each EV and the aggregator. Specifically, the sub-problem for each EV is

$$
\min _{x_{i, d}} C_{i, d}\left(x_{i, d}\right)-p_{m} x_{i, d}-\lambda x_{i, d} \quad \text { s.t. } 0 \leq x_{i, d} \leq h_{i, d},
$$

while the sub-problem for the aggregator is

$$
\min _{q} \quad D_{s}(q)+\lambda(G-q) \quad \text { s.t. } \quad 0 \leq q \leq G .
$$

In (3), by interpreting $\lambda$ as $p_{d}$, the unit price of regulation down, the optimization problem is to minimize the $i$-th EV's effective cost, which aligns exactly with the EV's interest. By the Karush-Kuhn-Tucker (KKT) conditions, given $\lambda$, the unique solution of the $i$-th EV is $\left[C_{i, d}^{\prime-1}\left(p_{m}+\lambda\right)\right]_{0}^{h_{i, d}}$. In (4), the aggregator aims to minimize its cost, including both the external energy cost and the payment to all EVs. Given $\lambda$, the unique solution of the aggregator is $\left[D_{s}^{\prime-1}(\lambda)\right]_{0}^{G}$.

The dual problem is defined as the maximization of the dual function:

$$
\max _{\lambda} g(\lambda) .
$$

Denote the optimal solution of the dual problem as $\lambda^{*}$ and the optimal value as $g^{*}$. Since Slater's condition is satisfied in P2, we have strong duality, i.e., $g^{*}=f^{*}$ [13]. In addition, since for any given $\lambda$, there is a unique solution in both (3) and (4), the dual function $g(\lambda)$ is everywhere continuously differentiable in $\mathbb{R}$, and the optimal point $\left(\mathbf{x}_{d}^{*}, q^{*}\right)$ of $\mathbf{P} 2$ can be recovered by solving (3) and (4) at the optimal dual solution $\lambda^{*}$ [18]. Applying the gradient method to the dual problem, we provide a distributed algorithm to derive $\lambda^{*}$ and $\left(\mathbf{x}_{d}^{*}, q^{*}\right)$, shown in Algorithm 1.

\section{B. Performance Analysis}

We first give the following lemma, which will be used for the convergence analysis of Algorithm 1.

Lemma 1: Suppose that the conditions $\mathbf{C 1}$ and $\mathbf{C 2}$ hold. Then the gradient of the dual function is Lipschitz continuous, i.e., $\left|g^{\prime}\left(\lambda_{1}\right)-g^{\prime}\left(\lambda_{2}\right)\right| \leq l\left|\lambda_{1}-\lambda_{2}\right|$ for all $\lambda_{1}, \lambda_{2} \in \mathbb{R}$, where

$$
l \triangleq(N+1) \max \left\{1 / c_{1, d}, \cdots, 1 / c_{N, d}, 1 / d_{s}\right\} .
$$

Proof: See our technical report [19].

Using Lemma 1, the convergence condition of Algorithm 1 is given as follows.

Theorem 1: Suppose that the conditions $\mathbf{C 1}$ and $\mathbf{C 2}$ hold. In Algorithm 1, if the step size $r \in\left(0, r_{0}\right)$ where $r_{0} \triangleq 2 / l$, then the generated $\left\{\lambda^{k}\right\}$ converges to the optimal dual solution $\lambda^{*}$.

Proof: Algorithm 1 is a specific case of the gradient algorithm. We refer readers to [18] for the detailed proof.

Remarks: From Theorem 1, the fixed step size $r$ is upper bounded by $r_{0}$, which is inversely proportional to the number of EVs and is related to all cost functions for providing regulation service. Therefore, roughly speaking, the larger the $\overline{\text { Algorithm } 1 \text { Synchronous distributed regulation allocation for }}$ regulation down.

Aggregator's algorithm:

1) Initialize the iteration index $k$ to 0 and the regulation price $\lambda^{0}$ to an arbitrary positive constant. If $\mathbf{1}_{d}=1$, broadcast to all EVs that the current service is regulation down.

2) At each iteration $k \in \mathbb{Z}^{+}$, broadcast the price signal $p_{d}=\lambda^{k}$ to all EVs, receive $x_{i, d}^{k}$ from each EV, derive the optimal amount of energy surplus by $q^{k}=\left[D_{s}^{\prime-1}\left(\lambda^{k}\right)\right]_{0}^{G}$, and update the price signal by

$$
\lambda^{k+1} \leftarrow \lambda^{k}+r\left(G-\sum_{i=1}^{N} x_{i, d}^{k}-q^{k}\right),
$$

where $r$ is a fixed step size.

3) Iteration terminates when the absolute value of the gradient $\left|G-\sum_{i=1}^{N} x_{i, d}^{k}-q^{k}\right|<\epsilon$, for some predefined $\epsilon>0$.

EV's algorithm:

1) Set $\mathbf{1}_{d}=1$ if the current service is regulation down.

2) At each iteration $k \in \mathbb{Z}^{+}$, receive the price signal $p_{d}$ from the aggregator, derive the optimal regulation amount by $x_{i, d}^{k}=\left[C_{i, d}^{\prime-1}\left(p_{m}+p_{d}\right)\right]_{0}^{h_{i, d}}$, and submit $x_{i, d}^{k}$ to the aggregator.

number of EVs, the slower the algorithm. Suppose that the batteries and the external energy source all have quadratic cost functions in the form of $c_{i, d} x^{2}$ and $d_{s} x^{2}$, respectively. Assume that the external source is more expensive, i.e., $d_{s}>c_{i, d}, \forall i$. Then, based on the definition of $l$ in Lemma 1, the estimate of the cost coefficient associated with the least-cost battery is required for the aggregator to determine the upper bound $r_{0}$.

Since EVs have energy gain by performing regulation down, the price signal could be negative when EVs' degradation costs are small. In the following proposition, we give a condition under which the price signal is lower bounded and there is an $\mathrm{EV}$ willing to perform regulation down.

Proposition 1: If there exists an $\mathrm{EV} j$ such that $C_{j, d}^{\prime}(x)-$ $p_{m}<D_{s}^{\prime}(x), \forall x \in(0, \eta)$, where $\eta$ is an arbitrarily small positive number, then $\lambda^{*}>\min _{1 \leq i \leq N}\left\{C_{i, d}^{\prime}(0)-p_{m}\right\}$ and $x_{j, d}^{*}>0$.

Proof: See technical report [19].

Proposition 1 essentially says that, as long as there is one EV whose effective marginal cost is strictly less than the marginal cost of the external energy source, it's beneficial for the aggregator to incentivize EVs to perform regulation service (although the price signal can be negative).

\section{Asynchronous Distributed Regulation ALLOCATION}

To implement Algorithm 1, the aggregator and all EVs are required to update the information at every iteration, which is impractical especially when the number of EVs is large. In addition, due to the communication delay between the aggregator and each EV, the price signal at each EV or the total regulation amount at the aggregator can be outdated. In this 
section, we modify Algorithm 1 and provide an asynchronous distributed algorithm for the aggregator-EVs system providing regulation service. Our approach is adapted from the general partially asynchronous method [20].

Denote $a_{i}$ as the communication delay from the aggregator to the $i$-th $\mathrm{EV}$, and $b_{i}$ as the communication delay from the $i$-th EV to the aggregator. Denote $\mathcal{T}_{i} \subseteq \mathbb{Z}^{+}$as the set of the iteration indexes for which the $i$-th EV updates its regulation amount. Specifically, if $k \in \mathcal{T}_{i}$, then the $i$-th EV updates $x_{i, d}^{k}$ based on the last received regulation price $p_{d}=\hat{\lambda}^{k}$; if $k \notin \mathcal{T}_{i}$, then the $i$-th EV skips this iteration, and its regulation amount at the aggregator remains unchanged. Similarly, denote $\mathcal{T}_{a} \subseteq$ $\mathbb{Z}^{+}$as the set of the iteration indexes for which the aggregator broadcasts and updates its information. In particular, if $k \in \mathcal{T}_{a}$, then the aggregator broadcasts $\lambda^{k}$, derives the energy surplus amount $q^{k}$, and updates the regulation price $\lambda^{k+1}$ based on the last received regulation amount from each $\mathrm{EV}$; if $k \notin \mathcal{T}_{a}$, then the aggregator skips this iteration and keeps the price unchanged. We assume the following partially asynchronous conditions [20]:

\section{C3:}

- The communication delays $a_{i}$ and $b_{i}, \forall i$, are bounded by a positive integer $d$.

- The aggregator and each EV update their information at least once every $d$ iterations.

Under the condition $\mathbf{C 3}$, we state the asynchronous distributed regulation allocation in Algorithm 2. Algorithm 2 is similar to the asynchronous algorithm presented in [21] for network flow control. Different from our model, the objective in [21] is to maximize the aggregate utility function of all sources subject to link capacities.

The convergence condition of Algorithm 2 is given below.

Theorem 2: Suppose that the conditions C1-C3 hold. Then, there exists a positive number $r_{\max }>0$ such that, if $r \in$ $\left(0, r_{\max }\right)$, then the generated $\left\{\lambda^{k}\right\}$ in Algorithm 2 converges to the optimal dual solution $\lambda^{*}$.

Proof: The proof is similar to that in [21]. The key is to show that the gradient of the dual function in (5) maintains in the ascent direction. See technical report [19].

\section{Simulation Results}

We have simulated an aggregator-EVs system with parameters drawn from practical scenarios, implementing Algorithms 1 and 2 to validate the theoretical results.

Suppose that the aggregator coordinates $N=100 \mathrm{EVs}$, evenly split into Type I (Ford Focus Electric) and Type II (Tesla Model S), to provide regulation service. For Type I EVs, we have $s_{i \text {,cap }}=23 \mathrm{kWh}$ and $x_{i, \max }=0.55 \mathrm{kWh}$; for Type II

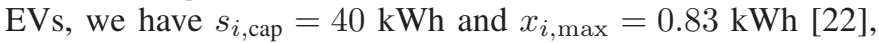
[23], where $x_{i, \max }$ for each $\mathrm{EV}$ is derived by assuming the regulation interval $\Delta t=5$ minutes. Assume that the current service is regulation down, and the regulation signal $G=69.2$ $\mathrm{kWh}$, which is the maximum allowed energy amount from all EVs. For each EV, we set $s_{i, \min }=0.1 s_{i, \text { cap }}$ and $s_{i, \max }=$ $0.9 s_{i, \text { cap }}$, and draw the initial energy state, $s_{i, b}$, uniformly from $\overline{\text { Algorithm } 2 \text { Asynchronous distributed regulation allocation }}$ for regulation down.

Aggregator's algorithm:

1) Initialize the iteration index $k$ to 0 and the regulation price $\lambda^{0}$ to an arbitrary positive constant. If $\mathbf{1}_{d}=1$, broadcast to all EVs that the current service is regulation down.

2) From time to time, receive the regulation amount from each EV.

3) At each iteration $k \in \mathcal{T}_{a}$ or $k=0$, broadcast the price signal $p_{d}=\lambda^{k}$ to all EVs, derive the optimal amount of energy surplus by $q^{k}=\left[D_{s}^{\prime-1}\left(\lambda^{k}\right)\right]_{0}^{G}$, and update the price by

$$
\lambda^{k+1} \leftarrow \lambda^{k}+r\left(G-\sum_{i=1}^{N} \hat{x}_{i, d}^{k}-q^{k}\right),
$$

where $r$ is a fixed step size, and $\hat{x}_{i, d}^{k}$ is the last received regulation amount from the $i$-th EV.

4) At each iteration $k \notin \mathcal{T}_{a}$, set $\lambda^{k+1} \leftarrow \lambda^{k}$.

5) Iteration terminates when the absolute value of the gradient $\left|G-\sum_{i=1}^{N} \hat{x}_{i, d}^{k}-q^{k}\right|<\epsilon$, for some predefined $\epsilon>0$.

EV's algorithm:

1) Set $\mathbf{1}_{d}=1$ if the current service is regulation down.

2) From time to time, receive the price signal from the aggregator.

3) At each iteration $k \in \mathcal{T}_{i}$, derive the optimal regulation amount based on the last received price $p_{d}=\hat{\lambda}^{k}$ by $x_{i, d}^{k}=\left[C_{i, d}^{\prime-1}\left(p_{m}+p_{d}\right)\right]_{0}^{h_{i, d}}$, and submit $x_{i, d}^{k}$ to the aggregator.

$\left[s_{i, \min }, s_{i, \max }\right]$. The charging efficiency coefficient is $\eta_{i, \mathrm{ch}}=$ 0.8 . The market charging price $p_{m}$ is 0.12 dollars $/ \mathrm{kWh}$. The degradation cost functions of Type I and Type II EVs are $0.1 x^{2}$ and $0.15 x^{2}$, respectively. The external cost function for clearing energy surplus is $0.2 x^{2}$. Hence, from Theorem 1 , the upper bound on a fixed step size in Algorithm 1 is $r_{0}=$ 0.002. In Algorithm 2, we set $\mathcal{T}_{i}=\cup_{k=0}\{2 k\}$ for Type I EVs, $\mathcal{T}_{i}=\cup_{k=1}\{2 k-1\}$ for Type II EVs, and $\mathcal{T}_{a}=\mathbb{Z}^{+}$for the aggregator. The bidirectional communication delay between the aggregator and each $\mathrm{EV}$ is 1, i.e., $a_{i}=b_{i}=1, \forall i$.

In Fig. 2, we exhibit the trace of the price signal $\left\{\lambda^{k}\right\}$ generated by Algorithm 1, where the step size $r$ is set as $0.1 r_{0}$, $r_{0}$, and $10 r_{0}$. We see that, for the first two step sizes, the price signal converges to the limit point 0.128 , while for the last step size, the price signal oscillates around 0.128 . This observation confirms the sufficient condition of $r$, i.e., $r \in\left(0, r_{0}\right)$, for the convergence of $\left\{\lambda^{k}\right\}$, and also demonstrates that, the convergence cannot be guaranteed if the step size is too large. In Table I, we list the required number of iterations for termination in Algorithm 1, where the termination condition is set as $\left|G-\sum_{i=1}^{N} x_{i, d}^{k}-q^{k}\right|<0.001$. The step size varies from $0.1 r_{0}$ to $r_{0}$. As expected, the required number of iterations decreases with the step size. In particular, for $r=0.1 r_{0}$ and $r_{0}$, the numbers of iterations are 299 and 26, respectively. 


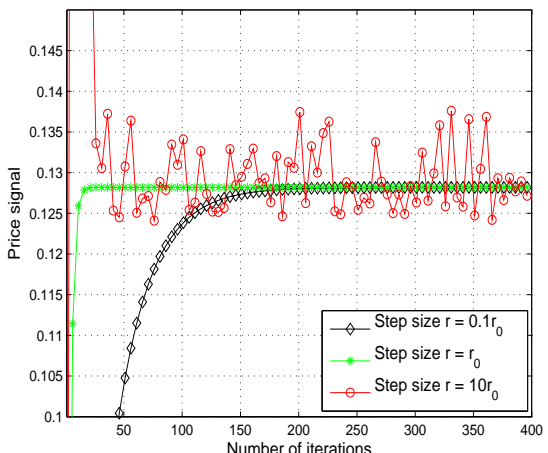

Fig. 2. Trace of the price signal $\left\{\lambda^{k}\right\}$ in Algorithm 1, with the step size $r=0.1 r_{0}, r_{0}$, and $10 r_{0}$.

TABLE I

REQUIRED NUMBER OF ITERATIONS FOR TERMINATION IN ALGORITHM 1.

\begin{tabular}{l|l|l|l|l|l|l|l}
\hline$r / r_{0}$ & 0.1 & 0.2 & 0.3 & 0.4 & 0.6 & 0.8 & 1 \\
\hline Number of iterations & 299 & 148 & 97 & 72 & 47 & 34 & 26 \\
\hline
\end{tabular}

This demonstrates the significance of the upper bound $r_{0}$, and hence the importance of estimating $l$, as defined in Lemma 1, in practice.

For the convergence of Algorithm 2, Theorem 2 provides a sufficient condition of the step size given by $0<r<r_{\max }$. We run Algorithm 2 with $r$ ranging from $0.1 r_{0}$ to $r_{0}$. Our results suggest that the price signal $\left\{\lambda^{k}\right\}$ converges to the same limit point for all these step sizes. Furthermore, in Fig. 3 , we show the trace of the dual function in (2). We see that, for $r$ ranging from $0.1 r_{0}$ to $0.2 r_{0}$, the dual function increases in every iteration, i.e., the price update is maintained in the ascent direction, while for $r \geq 0.3 r_{0}$, the ascent direction is not guaranteed. Therefore, in this example, for Algorithm 2 to be a gradient ascent algorithm, it suffices to set $r_{\max }=0.2 r_{0}$. However, we note that, although for $r \geq 0.3 r_{0}$ the ascent direction is not maintained, the convergence of $\left\{\lambda^{k}\right\}$ can still be used for the recovery of the optimal solution $\left(\mathbf{x}_{d}^{*}, q^{*}\right)$ in $\mathbf{P 2}$, indicating the robustness of the algorithm to the step size.

\section{CONCLUSION}

We considered an aggregator-EVs system performing regulation service, and developed synchronous and asynchronous distributed algorithms for regulation allocation. Our algorithms align each EV's interest with the system's benefit, and accommodate a more realistic aggregator-EVs system. For both synchronous and asynchronous algorithms, we provide sufficient conditions for optimality. The simulation results are exhibited to confirm the theoretical results.

\section{REFERENCES}

[1] B. Kirby, "Frequency regulation basics and trends," U.S. Dept. Energy, Tech. Rep., 2005.

[2] W. Kempton, V. Udo, K. Huber, K. Komara, S. Letendre, S. Baker, D. Brunner, and N. Pearre, "A test of vehicle-to-grid (V2G) for energy storage and frequency regulation in the PJM system," Tech. Rep., Nov. 2008. [Online]. Available: http://www.udel.edu/V2G/resources/ test-v2g-in-pjm-jan09.pdf

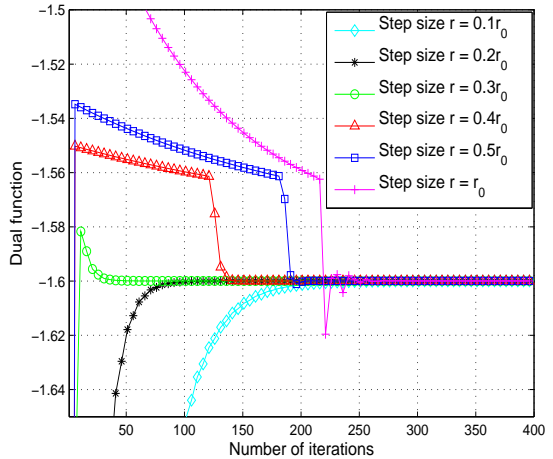

Fig. 3. Trace of the dual function in Algorithm 2, with various step sizes.

[3] R. Bessa and M. Matos, "Economic and technical management of an aggregation agent for electric vehicles: a literature survey," Eur. Trans. Elect. Power, vol. 22, pp. 334-350, Apr. 2011.

[4] D. Hill, A. Agarwal, and F. Ayello, "Fleet operator risks for using fleets for V2G regulation," ENERG POLICY, vol. 41, pp. 221-231, Feb. 2012.

[5] J. Garzas, A. Armada, and G. Granados, "Fair design of plug-in electric vehicles aggregator for $\mathrm{V} 2 \mathrm{G}$ regulation," IEEE Trans. Veh. Technol., vol. 61, pp. 3406-3419, Oct. 2012.

[6] S. Han, S. Han, and K. Sezaki, "Optimal control of the plug-in electric vehicles for V2G frequency regulation using quadratic programming," in Proc. IEEE ISGT, Jan. 2011.

[7] E. Sortomme and M. Sharkawi, "Optimal scheduling of vehicle-to-grid energy and ancillary services," IEEE Trans. Smart Grid, vol. 3, pp. 351359, Mar. 2012.

[8] S. Han, S. Han, and K. Sezaki, "Development of an optimal vehicleto-grid aggregator for frequency regulation," IEEE Trans. Smart Grid, vol. 1, pp. 65-72, Jun. 2010.

[9] W. Shi and V. Wong, "Real-time vehicle-to-grid control algorithm under price uncertainty," in Proc. IEEE SmartGridComm, Oct. 2011.

[10] S. Sun, M. Dong, and B. Liang, "Real-time welfare-maximizing regulation allocation in aggregator-EVs systems," in Proc. IEEE INFOCOM Workshop on CCSES, Apr. 2013.

[11] C. Wu, H. Rad, and J. Huang, "Vehicle-to-aggregator interaction game," IEEE Trans. Smart Grid, vol. 3, pp. 434-441, Mar. 2012.

[12] B. Gharesifard, T. Başar, and A. Domínguez-García, "Price-based distributed control for networked plug-in electric vehicles," in Proc. ACC, Jun. 2013.

[13] S. Boyd and L. Vandenberghe, Convex optimization. Cambridge University Press, 2004.

[14] D. Palomar and M. Chiang, "A tutorial on decomposition methods for network utility maximization," IEEE J. Sel. Areas Commun., vol. 24 pp. 1439-1451, Aug. 2006.

[15] S. Han, S. Han, and K. Sezaki, "Economic assessment on V2G frequency regulation regarding the battery degradation," in Proc. IEEE ISGT, Jan. 2012.

[16] P. Ramadass, B. Haran, R. White, and B. Popov, "Performance study of commercial LiCoO2 and spinel-based Li-ion cells," J. Power Sources, vol. 111, pp. 210-220, Apr. 2002.

[17] W. Kempton and J. Tomic, "Vehicle-to-grid power fundamentals: calculating capacity and net revenue," J. Power Sources, vol. 144, pp. 268279, Jun. 2005.

[18] D. Bertsekas, Nonlinear programming. Athena Scientific, 1999.

[19] S. Sun, M. Dong, and B. Liang, "Distributed regulation allocation with aggregator coordinated electric vehicles," University of Toronto, Tech. Rep., 2013. [Online]. Available: http://www.comm.utoronto.ca/ liang/ publications/techreport/distr_aggEV.pdf

[20] D. Bertsekas and J. Tsitsiklis, Parallel and Distributed Computation. Prentice-Hall, 1989.

[21] S. Low and D. Lapsley, "Optimization flow control - I: basic algorithm and convergence," IEEE/ACM Trans. Netw., vol. 7, pp. 861-874, Dec. 1999.

[22] Tesla Model S. [Online]. Available: http://www.teslamotors.com/

[23] Ford Focus Electric. [Online]. Available: http://www.ford.ca/cars/focus/ 